\title{
Multiwavelength Distribution Networks
}

\author{
Hung Q. Ngo \\ Computer Science and Engineering, \\ State University of New York at Buffalo. \\ hungngodcse.buffalo.edu
}

\begin{abstract}
We propose a graph model for the qualitative and quantitative analyses of wavelength division multiplexed (WDM) multicast/broadcast switching networks. The graph model, called $[w, f]$-distributor, turns out to be a generalized version of the graph model for classical circuit switching theory, which include important classes of graphs such as the distributors (also called generalized connectors). We then give lower bounds for $[w, f]$ distributors and $[w, f]$-distributors of a fixed depth. Many of the results generalize corresponding results from circuit switching. We also show that, under two prominent request models, strictly nonblocking $[w, f]$-distributors are equivalent.
\end{abstract}

\section{INTRODUCTION}

With the advances of dense wavelength division multiplexing (DWDM) technology [1-3], the number of wavelengths in a wavelength division multiplexed (WDM) network increases to hundreds or more per fiber, and each wavelength operates at 10Gbps (OC-192) or higher [4-6]. While raw bandwidth has increased by more than four order of magnitudes over the last decade or so, capacity of switches has only been up by a factor of ten [7]. Switching speed is the bottleneck at the core of the optical network infrastructure [7]. Consequently, a challenge is to design cost-effective photonic switching fabrics that can scale in size beyond a hundred of inputs and outputs, and at the same time, switch fast (e.g., tens of nanoseconds or less).

The notion of "cost-effectiveness" is difficult to capture. One can analyze and compare WDM switches both qualitatively and quantitatively.

Qualitatively, we need to know if a design is strictly nonblocking (SNB), rearrangeably nonblocking (RNB), and/or widesense nonblocking (WSNB) under different request models [8-12] and different traffic patterns (unicast [9], multicast [13]). Presumably each new design is guided by a particular qualitative feature. For example, one might come up with an RNB design under one request model, which may or may not be SNB under another request model. One might also have an intuitively good design, and hence need to know what qualitative feature the design possesses. This question is challenging in general. We shall see later that the graph models introduced in this paper help, in several ways, answer these types of questions.

Quantitatively, comparing different designs, or asking how close to be optimal a new design is, are very important questions. This is a multi-dimensional problem, as there are many factors effecting the "cost" of a switch. Some factors such as actual cost in dollars are business matters. Other factors include: the numbers of different types of switching components, cross-talk, power consumption and attenuation, integratability and scalability, blocking probabilities, and other factors such as the multicast capacity [13].

It should be apparent that we cannot hope to have a cost model that fits all needs. However, one can devise cost models which give good approximated measures on how "complex" a construction is. The notion of complexity should roughly capture as many practical parameters as possible.

In a recent paper, Ngo [14] outlined an intriguing approach to model switch complexity which not only helps analyze WDM switches quantitatively and qualitatively, but also suggests interesting generalizations of classical switching network theory $[15,16]$. Ngo then gave several complexity results and explicit constructions of the so-called $[w, f]$-connectors, which are graphs that model one-to-one WDM switching networks.

In this paper, we continue with the aforementioned approach and study graphs which model one-to-many WDM switching networks. We shall give several complexity bounds and an equivalence relationship of these graphs, some of which generalize known results in classical switching theory.

\section{REQUEST MODELS AND NONBLOCKINGNESS}

A general WDM cross-connect $\left(\mathrm{WXC}^{1}\right)$ consists of $f$ input fibers each of which can carry a set $\Lambda=\left\{\lambda_{1}, \ldots, \lambda_{w}\right\}$ of $w$ wavelengths, and $f^{\prime}$ output fibers each of which can carry a set $\Lambda^{\prime}=\left\{\lambda_{1}^{\prime}, \ldots, \lambda_{w^{\prime}}^{\prime}\right\}$ of $w^{\prime}$ wavelengths, where $f w=f^{\prime} w^{\prime}$. This setting is referred to as the heterogeneous case [11], which is needed to connect subnetworks from different manufacturers. From now on, let $n=f w=f^{\prime} w^{\prime}$, unless specified otherwise.

Let $\mathcal{F}=\left\{F_{1}, \ldots, F_{f}\right\}$ and $\mathcal{F}^{\prime}=\left\{F_{1}^{\prime}, \ldots, F_{f^{\prime}}^{\prime}\right\}$ denote the set of input and output fibers, respectively. There are many different request models for multicast WDM switching networks. We introduce here the two most common request models:

Request model 1: A multicast request is of the form $(\lambda, F, \mathcal{P})$ where $\lambda \in \Lambda, F \in \mathcal{F}$, and $\mathcal{P} \subseteq \Lambda^{\prime} \times \mathcal{F}^{\prime}$ such that no $F^{\prime} \in \mathcal{F}^{\prime}$ appears more than once in $\mathcal{P}$. (That is, if $(a, b)$ and $(c, d)$ are different pairs in $\mathcal{P}$, then $b \neq d$.) This restriction was made since in practical networks it is often not necessary to have a multicast connection going to the same output fiber on two different wavelengths $[13,17]$.

Request model 2: A multicast request is of the form $(\lambda, F, \mathcal{S})$, where $\lambda \in \Lambda, F \in \mathcal{F}$, and $\mathcal{S} \subseteq \mathcal{F}^{\prime}$. Basically, in this case we do not indicate the precise output wavelengths that the request should be routed to. We are only interested in the output

\footnotetext{
${ }^{1}$ We will use the term WXC to refer also to WDM switches where switching speed maybe fast enough for optical packet/burst switching.
} 
fibers $\mathcal{S}$. A multicast tree satisfying this request must have a leaf representing one wavelength from each fiber in $\mathcal{S}$.

For each type of request models, three degree of nonblockingness can be defined: rearrangeably nonblocking (RNB), wide-sense nonblocking (WSNB), and strictly nonblocking (SNB). The basic idea is that a RNB switching network should be able to route a set of compatible requests given in advance. In the WSNB case, requests are nonblocking provided that they are routed according to some algorithm. In the SNB case, a new request compatible with any valid network state can always be routed.

One might expect that the complexity a switching network could be less under model 2 than model 1, since nonblocking under model 1 implies nonblocking under 2. What is interesting is that this is not always the case, as we shall see later.

Due to space limitation, we have been informal in the descriptions above. The reader familiar with switching theory $[18,19]$ should not have difficulties understanding these concepts. We shall be more rigorous in our graph definitions to come.

\section{A GRAPH MODEL}

In this section, we describe a graph model proposed by $\mathrm{Ngo}$ [14], which was used to study one-to-one communication is WDM switching networks. We shall then extend this model to the one-to-many communication case.

We classify optical switching components into fibers and other switching components. For any switch design, we apply the following procedure to construct a directed acyclic graph (DAG) from the design: (a) replace each fiber by a set of vertices $\Lambda \cup \Lambda^{\prime}$, which represents all possible wavelengths which can be carried on the fiber; (b) the edges of the DAG are defined according to the functional capability of switching components in the design. The edges connect wavelengths (i.e. vertices) on the inputs of each switching component to the outputs in accordance with the functionality of the switching component.

Due to space limitation, we shall be brief on this construction. However, the reader will undoubtedly see the basic idea. As an example, Figure 1 shows how to turn an arrayed waveguide grating router (AWGR), a full-range wavelength converter (FWC), and a multiplexor (MUX) into edges. On the other

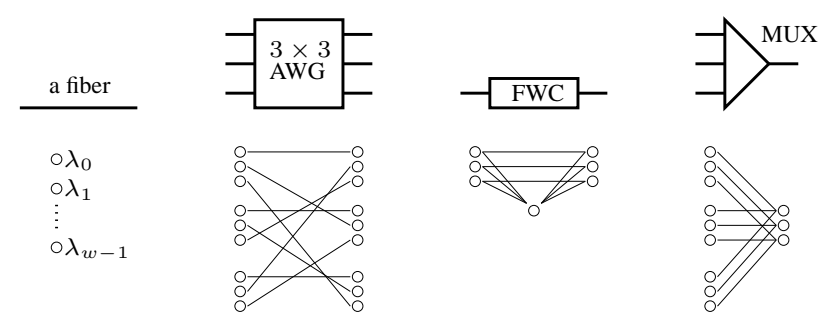

Fig. 1. Turning optical components into parts of a graph. A fiber is replaced by a set of vertices representing the wavelengths it can carry. Other components define edges connecting input wavelengths to output wavelengths. For the AWGR, MUX, and FWC, we illustrate with $w=3$. Edges are directed from left to right.

hand, Figure 2 shows a complete construction of the DAG from the design on the left. It is easy to see that a set of compatible
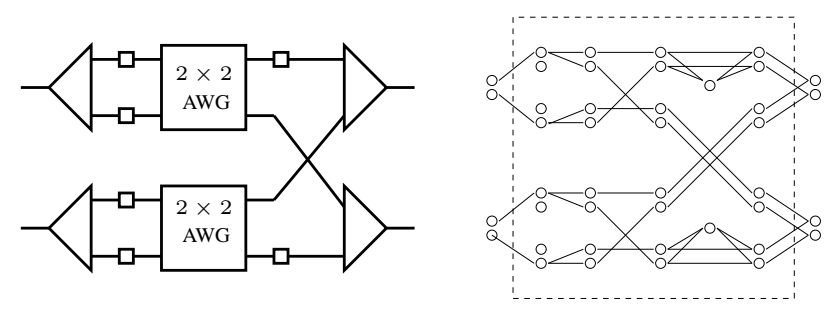

Fig. 2. A WDM switch design and its corresponding DAG.

routes from input wavelengths to output wavelengths correspond to a set of vertex disjoint paths from the inputs to the outputs of the DAG.

There are two main parameters of the DAG, which capture the notion of "switch complexity" discussed earlier.

The number of edges of the DAG, called the size of the DAG, is roughly proportional to the total cost of various components in the design. For example, a full-range wavelength converter (FWC) corresponds to $3 w$ edges while a wavelength interchanger [11] with corresponds to $w^{2}$ edges; a $w \times w$ AWGR corresponds to $w^{2}$ edges, a wavelength interchanger (WI) also corresponds to $w^{2}$ edges, while a $w \times w$ WDM crossbar corresponds to $w^{4}$ edges, etc. Since WIs and WDM crossbars are more expensive than FWCs and AWGRs, this model makes sense. Other components follow the same trend.

The reader might have noticed that different components contribute different "weights" to the total cost, hence summing up the number of edges may not give the "right" cost. To answer this doubt, we make three points. Firstly, as argued earlier one cannot hope to have a perfect model which fits all needs, and part of the notion of cost is a business matter. Our first aim is at a more theoretical level. Secondly, this is the first step toward a good cost model. One certainly can envision weighted graphs as the next step. Thirdly, we certainly can and should still use more traditional cost functions such as the direct counts of the number of each components and compare them individually.

The second measure on the DAG is its depth, i.e. the length of a longest path from any input to any output. As signals passing through different components of a design, they lose some power. The depth of the DAG hence reflects power loss, and in some cases even the signal delay. Again, different components impose different power loss factors. Hence, other information need to be taken into account to estimate power loss. However, it is clear that network depth is an important measure.

Last but not least, this DAG model provides a nice bridge between classical switching theory and WDM switching theory. As we shall see in later sections, this model helps us tremendously in answering qualitative questions about a particular construction. For example, if an $w f$-input $w f$-output DAG must have size $\Omega\left(f^{2} w^{2}\right)$ to be SNB, then we know for sure that a construction of cost (reflected by the DAG's size) $o\left(f^{2} w^{2}\right)$ cannot be SNB.

\section{More Rigorous SETtings}

In this section, we shall give more rigorous descriptions of the DAG models motivated from the last section. Our graphs shall capture different degrees of nonblockingness and the tradeoff between size and depth of a network. 
A $[w, f]$-network is a directed acyclic graph

$$
\mathcal{N}=\left(V, E ; A, B ; B_{1}, \ldots, B_{f}\right)
$$

with vertex set $V$ and edge set $E$, a set $A$ of $n=w f$ distinguished nodes called inputs and a disjoint set $B$ of $n$ distinguished nodes called outputs. The set $B$ is further partitioned into $f$ subsets $B_{1}, \ldots, B_{f}$ of size $w$ each. The sets $B_{i}$ represent output fibers, and their elements represent wavelengths. To shorten notations, we shall write $\mathcal{N}=(V, E ; A, B)$, with the $B_{i}$ being implicit. The vertices in $V-A \cup B$ are called internal vertices. The in-degrees of the inputs and the out-degrees of the outputs are zero. The size of a network is its number of edges. The depth of a network is the maximum length of a path from an input to an output. Note that we do not specify input fibers and their wavelengths since they are indistinguishable as far as our request models are concerned.

\section{(a) Request model 1}

For any set $X$, let $P(X)$ denote the power set of $X$. Given a $[w, f]$-network $\mathcal{N}=(V, E ; A, B)$, a pair $D=(a, S) \in A \times$ $P(B)$, where $\left|S \cap B_{i}\right| \leq 1, \forall i$, is called a distribution request under model $1\left(D R_{1}\right)$ for $\mathcal{N}$. As we are only concerned with distribution networks in this paper, the term "request" should be implicitly understood as "distribution request" henceforth.

A distribution assignment under model $l\left(D A_{1}\right)$ is a set $\mathcal{D}$ of $D R_{1}$ where no two requests share an input nor an output. A $D R_{1} D=(a, S)$ is compatible with a $D A_{1} \mathcal{D}$ iff $\mathcal{D} \cup\{D\}$ is also a $D A_{1}$. A distribution route $R$ for a $D R_{1} D=(a, S)$ is a (directed) tree rooted at $a$ whose leaves are nodes in $S$. We also say $R$ realizes $D$. A state of $\mathcal{N}$ is a set $\mathcal{R}$ of vertex disjoint distribution routes. Each state of $\mathcal{N}$ realizes a unique $D A_{1}$, one route per request. A $D A_{1} \mathcal{D}$ is realizable iff there is a network state realizing it. A request is compatible with a state if it is compatible with the $D A_{1}$ realized by the state.

A rearrangeable $(R N B)[w, f]_{1}$-distributor is a $[w, f]$ network in which any $D A_{1}$ is realizable.

A strictly nonblocking $(S N B)[w, f]_{1}$-distributor is a $[w, f]$ network $\mathcal{N}$ in which given any network state $\mathcal{R}$ realizing a $D A_{1}$ $\mathcal{D}$, and given a new request $D$ compatible with $\mathcal{D}$, there exists a route $R$ such that $\mathcal{R} \cup\{R\}$ is a network state realizing $\mathcal{D} \cup\{D\}$.

As requests come and go, a strategy to pick new routes for new requests is called a routing algorithm. A $[w, f]$-network is called a widesense nonblocking (WSNB) $[w, f]_{1}$-distributor with respect to a routing algorithm $\mathbf{A}$ if $\mathbf{A}$ can always pick a new route for a new request compatible with the current network state. We can also replace $\mathrm{A}$ by a class of algorithms $\mathcal{A}$. In general, an $[w, f]$-network $\mathcal{N}$ is WSNB iff it is WSNB with respect to some algorithm.

We often consider two classes of functions on each network type: (a) the minimum size of a network, and (b) the minimum size of a network with a given depth. The main theme of research on classical switching networks is to investigate the tradeoffs between size and depth [16,20].

Let $r d_{1}(w, f), w d_{1}(w, f)$, and $s d_{1}(w, f)$ denote the minimum size of an RNB, WSNB, and SNB $[w, f]_{1}$-distributor, respectively. Let $r d_{1}(w, f, k), w d_{1}(w, f, k)$, and $s d_{1}(w, f, k)$ denote the minimum size of an RNB, WSNB, and SNB $[w, f]_{1^{-}}$ distributor with depth $k$, respectively.
Remark IV.1. In the classical switching literature, distributors are also called generalized connectors.

\section{(b) Request model 2}

The difference between model 2 and model 1 is that a request only specify the set of output fibers, not the specific wavelengths on the fibers.

Given a $[w, f]$-network $\mathcal{N}$, a distribution request under model $2\left(D R_{2}\right)$ is a pair $D=(a, T) \in A \times P(\{1, \ldots, f\})$. A distribution route $R$ for a $D R_{2} D=(a, T)$ is a tree rooted at $a$ with exactly $|T|$ leaves, one in each $B_{j}, j \in T$. A distribution assignment under model $2\left(D A_{2}\right)$ is a set $\mathcal{D}$ of $D R_{2}$ such that no two inputs appear twice in $\mathcal{D}$, and that for each $j \in\{1, \ldots, f\}$,

$$
\mid\{T:(a, T) \in \mathcal{D} \text { and } j \in T\} \mid \leq w .
$$

In words, no output fiber is involved in more than $w$ requests. The rest of the definitions are similar to the ones under model 1. The following observations are straightforward from definitions.

Proposition IV.2. $A n S N B, W S N B, R N B[w, f]_{1}$-distributor is also an $S N B, W S N B, R N B[w, f]_{2}$-distributor, respectively. Consequently, $\mathbf{x} d_{2}(\cdot) \leq \mathbf{x} d_{1}(\cdot)$, where $\mathbf{x}$ stands for either $r, w$ or $s$, and the $\cdot$ is either $(w, f)$ or $(w, f, k)$.

Proposition IV.3. We have

$$
r d_{i}(\cdot) \leq w d_{i}(\cdot) \leq s d_{i}(\cdot),
$$

where $i=1,2$, and the $\cdot$ is either $(w, f)$ or $(w, f, k)$.

\section{ON THE EQUIVALENCE OF STRICTLY NONBLOCKING $[w, f]$-DISTRIBUTORS UNDER TWO REQUEST MODELS}

The following theorem essentially shows that being SNB in the more relaxed request model 2 gives us no advantage as far as network cost is concerned.

Theorem V.1. Let $w, f$ be positive integers where $f \geq 2$. Then, $a[w, f]$-network is an $S N B[w, f]_{1}$-distributor if and only if it is an $\operatorname{SNB}[w, f]_{2}$-distributor.

Proof. It is obvious that an SNB $[w, f]_{1}$-distributor is also an SNB $[w, f]_{2}$-distributor. We now show the converse.

Let $\mathcal{N}=(V, E ; A, B)$ be an SNB $[w, f]_{2}$-distributor. (Recall that the partition $B=B_{1} \cup \cdots \cup B_{f}$ is implicit.) Let $\mathcal{R}$ be a state of $\mathcal{N}$, namely $\mathcal{R}$ is a set of vertex disjoint trees whose roots are inputs, whose leaves are outputs of $\mathcal{N}$. Let $D=(a, S)$ be a $D R_{1}$ compatible with $\mathcal{R}$. We shall show that there is a tree $R$ rooted at $a$ with leaves $S$, and $R$ is vertex disjoint from trees in $\mathcal{R}$. For each $s \in S$, let $B_{j(s)}$ denote the output band in which $s$ is a member of. Recall that, by definition of $D R_{1}$, $B_{j(s)} \neq B_{j\left(s^{\prime}\right)}$, for members $s \neq s^{\prime}$ of $S$.

The main idea is that we shall show there is a state $\mathcal{S}$ of $\mathcal{N}$ such that $\mathcal{R} \subseteq \mathcal{S}, a$ is free in $\mathcal{S}$, and that each $s$ in $S$ is the only free output in $B_{j(s)}$. Suppose such a state $\mathcal{S}$ can be constructed. Consider the request $(a, T)$, where $T=\{j(s) \mid s \in S\}$. This request is compatible with $\mathcal{S}$ under model 2. Since $\mathcal{N}$ is an SNB $[w, f]_{2}$-distributor, there is a tree $R$ realizing $(a, T)$. This 
is the tree we are looking for, as the leaves of the tree have to be precisely those in $S$.

To show the existence of such a state $\mathcal{S}$, let us consider two cases as follows.

Case 1: there is some route in $\mathcal{R}$ with more than one leaf. Let $X$ $(Y)$ be the number of free inputs (outputs) in $\mathcal{R}$. Then, $a \in X$ and $|X|>|Y|$, because the total numbers of inputs and outputs are the same. Now, let $k$ be such that $B_{k}$ has some free output in $\mathcal{R}$. Let $x$ be a member of $X-\{a\}$. The request $(x, k)$ is compatible with $\mathcal{R}$; hence, there is a route $R_{1}$ from $x$ to some output in $B_{k}$ for which $\mathcal{R} \cup\left\{R_{1}\right\}$ is a state. Repeat this process $|Y|$ times, we will have a state $\mathcal{R}^{\prime}=\mathcal{R} \cup\left\{R_{1}, \ldots, R_{|Y|}\right\}$ in which there is no more free outputs, yet $a$ is still free. Now, remove from $\mathcal{R}^{\prime}$ all routes whose endpoints are those in $S$, we get the desired state $\mathcal{S}$.

Case 2: all routes in $\mathcal{R}$ are one-to-one routes. This is a much trickier case, as $|X|=|Y|$ and $a$ has to be involved in the "filling up" process. As in case 1, we make requests of the form $(x, k), x \in X$. The vertex $a$ is somewhat special, we make sure that a request $(a, j(\bar{s}))$ was created first, for some $\bar{s} \in S$. The rest of the $(x, k)$ requests are arbitrary as before. For each $x \in X$, let $R_{x}$ denote the corresponding route for the request $(x, k)$. As in case 1 , let $\mathcal{R}^{\prime}=\mathcal{R} \cup\left\{R_{x} \mid x \in X\right\}$ be the final state.

If $R_{a}$ ends at $\bar{s}$, then we are lucky. Remove from $\mathcal{R}^{\prime}$ the routes $R_{a}$ and all the $R_{x}$ which end at some $s$ in $S$, we get the desired state $\mathcal{S}$.

If we are not lucky, $R_{a}$ ends at some $t$ in $B_{j(\bar{s})}, t \neq \bar{s}$. Let $\bar{a} \in X$ be an input such that $R_{\bar{a}}$ ends at $\bar{s}$. Let $b$ be any input whose corresponding route in $\mathcal{R}^{\prime}$ is $\left(b, v_{1}, \ldots, v_{p}, u\right)$, where $u \in B_{i}$ for some $i \neq j(\bar{s})$. (Since $f \geq 2$, we are sure that there is some $i \neq j(\bar{s})$.)

Let $\mathcal{S}^{\prime}=\mathcal{R}^{\prime}-\left\{R_{a}, R_{\bar{a}}, R_{b}\right\}$, which is a network state.

We claim that there is an $(\bar{a}, t)$-route compatible with $\mathcal{S}^{\prime}$. Consider the state $\mathcal{S}^{\prime} \cup\left\{R_{a}\right\}$. The request $(b, j(\bar{s}))$ is compatible with the state. Moreover, $\bar{s}$ is the only free output in $B_{j(\bar{s})}$ in the state $\mathcal{S}^{\prime}$. Thus, there is a $(b, \bar{s})$-route $R_{b \bar{s}}$ such that $\mathcal{S}^{\prime} \cup\left\{R_{a}, R_{b \bar{s}}\right\}$ is a state. Now, in the state $\mathcal{S}^{\prime} \cup\left\{R_{b \bar{s}}\right\}$ the output $t$ is the only free output in $B_{j(\bar{s})}$. Hence, the compatible request $(\bar{a}, j(\bar{s}))$ has to be routed to $t$. Hence, there is an $(\bar{a}, t)$-route compatible with $\mathcal{S}^{\prime}$ as claimed.

To this end, we further consider two cases as follows. (2a) among all $(\bar{a}, t)$-routes which are compatible with $\mathcal{S}^{\prime}$, there is a route $R_{\bar{a} t}$ which is vertex disjoint from $R_{b}$. In this case, $\mathcal{S}^{\prime} \cup\left\{R_{b}, R_{\bar{a} t}\right\}$ is a state in which $a$ and $\bar{s}$ are the only free vertices. A request $(a, j(\bar{s}))$ then brings us back to the "lucky" situation considered earlier.

(2b) every $(\bar{a}, t)$-route compatible with $\mathcal{S}^{\prime}$ intersects $R_{b}$ at some point. Let $R_{\bar{a} t}$ be such an $(\bar{a}, t)$-route whose last intersection vertex on $\left(v_{1}, \ldots, v_{p}\right)$ has the largest index, say $v_{q}$, where $1 \leq$ $q \leq p$. Then, $R_{\bar{a} t}$ is composed of two parts: the part from $\bar{a}$ to $v_{q}$, and the part from $v_{q}$ to $t$.

Now, let $R_{b t}$ be a $(b, t)$-path consisting of the part $\left(b, v_{1}, \ldots, v_{q}\right)$ concatenated with the $\left(v_{q}, t\right)$-part of $R_{\bar{a} t}$. Then, certainly $\mathcal{S}^{\prime} \cup\left\{R_{b t}\right\}$ is a state in which the request $(a, j(\bar{s}))$ is valid, and $\bar{s}$ is the only free output in $B_{j(\bar{s})}$. Hence, there is an $(a, \bar{s})$-route $R_{a \bar{s}}$ which is compatible with $\mathcal{S}^{\prime} \cup\left\{R_{b t}\right\}$.

If the route $R_{a \bar{s}}$ is vertex disjoint from $R_{b}$, then the request $(\bar{a}, j(\bar{s}))$ under the state $\mathcal{S}^{\prime} \cup\left\{R_{b}, R_{a \bar{s}}\right\}$ can only be satisfied by routing $\bar{a}$ to $t$. The resulting state brings us back to the "lucky" situation.

For the contrary, suppose $R_{a \bar{s}}$ intersects $R_{b}$ at some vertex. Then, due to the fact that $R_{a \bar{s}}$ is vertex disjoint from $R_{b t}$, the vertices in the intersection must all come after $v_{q}$. Let $v_{q^{\prime}}, q^{\prime}>$ $q$, be a vertex in the intersection of $R_{a \bar{s}}$ and $R_{b}$. Let $R_{b \bar{s}}$ be the route obtained by concatenating the route $\left(b, v_{1}, \ldots, v_{q^{\prime}}\right)$ and the $\left(v_{q^{\prime}}, \bar{s}\right)$-part of $R_{a \bar{s}}$. In the state $\mathcal{S}^{\prime} \cup\left\{R_{b \bar{s}}\right\}$, the request $(\bar{a}, j(\bar{s}))$ is valid. A route realizing this request must intersect $R_{b}$ (since we are in case $2 \mathrm{~b}$ ) at a point after $v_{q^{\prime}}$ (since we are in the state $\mathcal{S}^{\prime} \cup\left\{R_{b \bar{s}}\right\}$ ), contradicting the maximality of $q$.

Corollary V.2. Given positive integers $w, f$, and $k$, we have $s d_{1}(w, f)=s d_{2}(w, f)$ and $s d_{1}(w, f, k)=s d_{2}(w, f, k)$.

Corollary V.3. $s d_{1}(w, f, 1)=s d_{2}(w, f, 1)=(w f)^{2}$.

Proof. It is easy to see that $s d_{1}(w, f, 1)=(w f)^{2}$.

\section{LOWER BOUNDS FOR REARRANGEABLE $[w, f]$-DISTRIBUTORS}

An $n$-network is a directed acyclic graph $G=(V, E ; A, B)$ with a subset $A$ of $n$ vertices called inputs and a disjoint subset $B$ of $n$ vertices called outputs. The inputs (outputs) have indegree (out-degree) zero.

Let $A=\left\{a_{0}, \ldots, a_{n-1}\right\}$ and $B=\left\{b_{0}, \ldots, b_{n-1}\right\}$. An $n$ shifter is an $n$-network $G=(V, E ; A, B)$ such that for each $k \in\{0, \ldots, n-1\}$, there are $n$ vertex disjoint paths joining $a_{i}$ to $b_{(i+k) \bmod n}$, for $i=0, \ldots, n$.

The following lemma was shown by Pippenger and Yao [21].

Lemma VI.1. An $n$-shifter of depth $k$ has at least $k n^{1+\frac{1}{k}}$ edges.

Theorem VI.2. For $k \geq 2$, a depth- $k[w, f]_{1}$-distributor must have size at least $k(w f)^{1+1 / k}$. Specifically

$$
r d_{1}(w, f, k) \geq k(w f)^{1+1 / k} .
$$

Proof. Let $\mathcal{N}=(V, E ; A, B)$ be a depth- $k[w, f]_{1}$-distributor. Let $n=w f$. Arbitrarily assign labels to the inputs in $A$ and outputs in $B$ so that $A=\left\{a_{0}, \ldots, a_{n-1}\right\}$ and $B=$ $\left\{b_{0}, \ldots, b_{n-1}\right\}$. For each $q=0, \ldots, n-1$, consider the following set

$$
\mathcal{D}_{q}=\left\{\left(a_{i},\left\{b_{(i+q) \bmod n}\right\}\right) \mid i \in\{0, \ldots, n-1\}\right\} .
$$

Clearly $\mathcal{D}_{q}$ is a $D A_{1}$. Hence, there exists $n$ vertex disjoint paths joining $a_{i}$ to $b_{(i+q) \bmod n}$. Consequently, $\mathcal{N}$ is an $n$-shifter of depth $k$. Our result now follows from Lemma VI.1.

Corollary VI.3. For $k \geq 2, r d_{1}(w, f) \geq \operatorname{ewf}(\ln f+\ln w)$, where $e$ is the base of the natural log.

Proof. The function $g(k)=k(w f)^{1+1 / k}$, with $k \geq 1$, is minimized at $k=\ln (w f)$.

Let $T_{k}(f)$ be a directed rooted tree with $f$ leaves and depth at most $k$ where all edges directed to the direction of the leaves. Let $P_{1}, \ldots, P_{f}$ be the $f$ paths from the root to the leaves of $T_{k}(f)$. Define

$$
\Delta\left(T_{k}(f)\right):=\sum_{j=1}^{f} \sum_{v \in P_{j}} \text { out-degree }(v) .
$$


For the case of $[w, f]_{2}$-distributors, we shall make use of an idea and the following lemma from [21].

Lemma VI.4. $\Delta\left(T_{k}(f)\right) \geq k f^{1+\frac{1}{k}}$.

Theorem VI.5. For $k \geq 2$, a depth- $k[w, f]_{2}$-distributor must have size at least $k w f^{1+1 / k}$. Specifically

$$
r d_{2}(w, f, k) \geq k w f^{1+1 / k} \text {. }
$$

Proof. Note that we are working under request model 2. As usual, let $n=w f$. Let $\mathcal{N}=(V, E ; A, B)$ be a $[w, f]_{2^{-}}$ distributor, where $A=\left\{a_{1}, \ldots, a_{n}\right\}$. For each $q$ in $\{1, \ldots, f\}$, define a function

$$
\phi_{q}(i)=(i+q-1 \quad(\bmod f))+1,1 \leq i \leq n .
$$

Also define the following $D A_{2}$, for each $q$,

$$
\mathcal{D}_{q}:=\left\{\left(a_{i},\left\{\phi_{q}(i)\right\}\right) \mid 1 \leq i \leq n\right\} .
$$

Since $\mathcal{N}$ is a $[w, f]_{2}$-distributor, for each $q=1, \ldots, f$ there are $n$ vertex disjoint paths $P_{i q}, i=1, \ldots, n$, such that $P_{i q}$ joins $a_{i}$ to some vertex in $B_{\phi_{q}(i)}$.

To this end, for $1 \leq i \leq n, 1 \leq q \leq f$, and $e \in E$, let

$$
\mu(i, q, e):= \begin{cases}1 & \text { if } e \text { is an arc emitted from a node on } P_{i q} \\ 0 & \text { otherwise. }\end{cases}
$$

Fix an $i$, assemble all $f$ paths $P_{i q}$ into a tree $T_{i}$ (keeping only the initial common segments of the paths), then $T_{i}$ is a tree with $f$ leaves and depth at most $k$.

For each vertex $v \in V$, let out-degree $T_{T_{i}}(v)$ denote the outdegree of $v$ in $T_{i}$. It is easy to see the following

$$
\sum_{e \in E} \mu(i, q, e) \geq \sum_{v \in P_{i q}} \text { out-degree }_{T_{i}}(v) .
$$

Basically, the left hand side counts also some arcs not in $T_{i}$ (but starts on $P_{i q}$ ).

Summing (5) over $i=1, \ldots, n$ and $q=1, \ldots, f$, we get

$$
\begin{aligned}
\sum_{i=1}^{n} \sum_{q=1}^{f} \sum_{e \in E} \mu(i, q, e) & \geq \sum_{i=1}^{n} \sum_{q=1}^{f} \sum_{v \in P_{i q}} \text { out-degree }_{T_{i}}(v) \\
& =\sum_{i=1}^{n} \Delta\left(T_{i}\right) \\
& \geq n k f^{1+1 / k}
\end{aligned}
$$

The last inequality comes from Lemma (VI.4).

On the other hand, since the paths $P_{i q}$ for a fixed $q$ are vertex disjoint, we have

$$
\sum_{i=1}^{n} \mu(i, q, e) \leq 1
$$

Consequently,

$$
\sum_{i=1}^{n} \sum_{q=1}^{f} \sum_{e \in E} \mu(i, q, e)=\sum_{q=1}^{f} \sum_{e \in E} \sum_{i=1}^{n} \mu(i, q, e) \leq f|E| .
$$

Together, (6) and (7) lead to $|E| \geq k w f^{1+1 / k}$ as desired.

We get a similar result as the one in Corollary VI.3.

Corollary VI.6. For $k \geq 2, r d_{2}(w, f) \geq e w f \ln f$, where e is the base of the natural log.

\section{ACKNOWLEDGEMENTS}

Hung Q. Ngo is supported by NSF CAREER Award CCF0347565 .

\section{REFERENCES}

[1] Rajiv Ramaswami and Kumar Sivarajan, Optical Networks: A Practical Perspective (Second Edition), Morgan Kaufmann Publishers, San Francisco, CA, 2001.

[2] Thomas E. Stern and Kirshna Bala, Multiwavelength Optical Networks: A Layered Approach, Prentice Hall PTR, Upper Saddle River, NJ, 1999.

[3] B. Mukherjee, Optical Communication Networks, McGraw-Hill, New York, NY, 1997.

[4] Lucent Technologies Press Release, "Lucent Technologies unveils untra-high-capacity optical system; Time Warner Telecom first to announce it will deploy the system," 2001, http://www.lucent.com/press/0101/010117.nsa.html.

[5] Lucent Technologies Press Release, "Lucent Technologies engineer and scientists set new fiber optic transmission record," 2002, http://www.lucent.com/press/0302/020322.bla.html.

[6] Lucent Technologies Website, "What is dense wave division multiplexing (DWDM)," 2002, http://www.belllabs.com/technology/lightwave/dwdm.html.

[7] Amit Singhal and Raj Jain, "Terabit switching: a survey of techniques and current products," Computer communications, vol. 25, no. 8, pp. 547-556, 2002.

[8] G. Wilfong, B. Mikkelsen, C. Doerr, and M. Zirngibl, "WDM crossconnect architectures with reduced complexity," Journal of Lightwave Technology, vol. 17, no. 10, pp. 1732-1741, Oct 1999.

[9] Hung Q. Ngo, Dazhen Pan, and Chunming Qiao, "Nonblocking WDM switches based on arrayed waveguide grating and limited wavelength conversion," in Proceedings of the 23rd Conference of the IEEE Communications Society (INFOCOM'2004, HongKong). 2004, IEEE.

[10] April Rasala and Gordon Wilfong, "Strictly non-blocking WDM crossconnects," in Proceedings of the Eleventh Annual ACM-SIAM Symposium on Discrete Algorithms (SODA'2000, San Francisco, CA), New York, 2000, pp. 606-615, ACM.

[11] April Rasala and Gordon Wilfong, "Strictly non-blocking WDM crossconnects for heterogeneous networks," in Proceedings of the ThirtySecond Annual ACM Symposium on Theory of Computing (STOC'2000, Portland, OR), New York, 2000, pp. 513-524, ACM.

[12] Jeyashankher Ramamirtham and Jonathan S. Turner, "Design of wavelength converting switches for optical burst switching," in Proceedings of the 21st Annual Joint Conference of the IEEE Computer and Communications Societies (INFOCOM). 2002, vol. 2, pp. 1162-1171, IEEE.

[13] Yuanyuan Yang, Jianchao Wang, and Chunming Qiao, "Nonblocking WDM multicast switching networks," IEEE Trans. Para. and Dist. Sys., vol. 11 , no. 12, pp. 1274-1287, Dec 2000

[14] Hung Q. Ngo, "WDM switching networks and $[w, f]$-connectors," 2004, Submitted to IPLCA' 2004.

[15] V. E. Beneš, Mathematical theory of connecting networks and telephone traffic, Academic Press, New York, 1965, Mathematics in Science and Engineering, Vol. 17.

[16] Nicholas Pippenger, "Communication networks," in Handbook of theoretical computer science, Vol. A, pp. 805-833. Elsevier, Amsterdam, 1990.

[17] Yang Wang and Yuanyuan Yang, "Multicasting in a class of multicastcapable WDM networks," J. Lightwave Technol., vol. 13, no. 2, pp. 128141, Feb 2002.

[18] Frank K. Hwang, The mathematical theory of nonblocking switching networks, World Scientific Publishing Co. Inc., River Edge, NJ, 1998.

[19] H. Hinton, An Introduction to Photonic Switching Fabrics, Plenum Press, New York, NY, 1993.

[20] Hung Q. Ngo and Ding-Zhu Du, "Notes on the complexity of switching networks," in Advances in Switching Networks, D.-Z. Du and Hung Q. Ngo, Eds., pp. 307-367. Kluwer Academic Publishers, 2001.

[21] Nicholas Pippenger and Andrew C. C. Yao, "Rearrangeable networks with limited depth," SIAM J. Algebraic Discrete Methods, vol. 3, no. 4, pp. 411-417, 1982 . 